\title{
Las estructuras cognitivas en la construcción de un modelo didáctico para Ambientes Virtuales de Aprendizaje (AVA)*
}

\section{Cognitive structures in the construction of a didactic model for Virtual Learning Environments (VLE)}

\section{As estruturas cognitivas na construção de um modelo didático para Ambientes Virtuais de aprendizagem (AVA)}

\author{
Jesús Salvador Moncada Cerón** \\ Universidad Antonio Nariño, Bogotá, Colombia
}

\begin{abstract}
Resumen. Hoy en día existe una gran variedad de ambientes virtuales de aprendizaje (AVA) que intentan dar respuesta a las demandas educativas actuales pero que, en muchos de los casos, resultan poco eficaces en el cumplimiento de las expectativas para los cuales fueron creados. El desarrollo de un AVA requiere de la definición de una serie de estrategias didácticas cognitivas, en relación a la planeación del acto educativo y de la expresión visual y formal del mismo, los cuales permitan definir modelos para su creación acordes con su eficacia en el cumplimiento de las intenciones educativas. Esta revisión busca apoyar con sus planteamientos la influencia de
\end{abstract}

* Artículo de avance de investigación dentro del proyecto de investigación denominado La metacognición en ambientes e-learning, desarrollado por el grupo de investigación Culturas Universitarias, de la Universidad Antonio Nariño, Bogotá, Colombia.

** Doctor en Educación Universidad La Salle México, Maestría en innovación educativa Universidad La Salle México, Decano de la Facultad de Educación Universidad Antonio Nariño, Bogotá, Colombia. e-mail: decano.educacion@uan.edu.co 
las estructuras cognitivas en la construcción de un modelo didáctico acorde a la identificación de los factores que determinan la creación de un AVA.

Palabras clave. AVA, educación, didáctica, cognición, constructivismo (Tesauro Unesco).

Abstract. Nowadays, there is a variety of virtual learning environments (VLE) attempting to respond to current educational demands, but in many cases, these are not very effective in meeting the expectations for which they were created. The development of VLE involves defining a series of didactic cognitive strategies related not only to formal teaching and learning plans, but also to visual aids and instructional design. This series allows the establishment of models for VLE's creation in accordance with its efficiency in meeting educational aims and objectives. With its proposals, this review seeks to support the influence of cognitive structures in the construction of a teaching model, according to the identification of the factors that determine the creation of a VLE.

Keywords. VLE, education, didactic, cognition, constructionism (Thesaurus Unesco).

Resumo. Hoje em dia existe uma grande variedade de ambientes virtuais de aprendizagem (AVA) que tentam responder as demandas educativas atuais, mas que, em muitos casos, resultam pouco eficazes na execução das expectativas para os quais foram criados. O desenvolvimento de um AVA requer a definição de uma série de estratégias didático- cognitivas, em relação ao planejamento do ato educativo e da expressão visual e formal do mesmo, para que os quais permitam definir modelos para que sua criação se conforme com sua eficácia no cumprimento das intenções educativas. Esta revisão busca apoiar com suas exposições a influência das estruturas cognitivas na construção de um modelo didático que concorde com a identificação dos fatores que determinam a criação de um AVA.

Palavras chave. AVA, educação, didática, cognição, construtivismo (Tesauro Unesco).

\section{Introducción}

Un ejercicio comprensivo acerca de las metodologías didácticas virtuales conocidas como Ambientes Virtuales de Aprendizaje (AVA) lo da una 
revisión juiciosa de los procesos cognitivos implícitos en su funcionalidad. En el presente artículo se intenta tal revisión con el propósito de explicitar la manera como las denominadas metodologías AVA, en su apuesta didáctica, están definidas claramente por una arquitectura cognitiva, que en la mayoría de los casos, no se interrelacionan directamente con la pedagogía que se les imprime. Es así que para Ginés (2005), la idea de la transformación de las universidades se puede sintetizar en un criterio por transformar el paradigma educativo sustentado en el conocimiento, a otro que dé prioridad a la formación integral de las personas. Se ha de enfatizar una atención específica al desarrollo de habilidades metodológicas, sociales y participativas, con la consigna de hacer pragmáticos los planteamientos teóricos. De igual manera, considera primordial que el modelo organizativo de las universidades debe proyectarse al incremento de la flexibilidad temporal del sistema y favorecer con ello la formación a lo largo de la vida y la integración del estudiante al ámbito laboral.

Lo anterior hace evidente la necesidad de revisar y transformar los procesos educativos que rigen a las universidades, en donde las llamadas tecnologías de la información y la comunicación jugarán un papel decisivo para el desarrollo e implementación de dichos procesos ante las nuevas demandas productivas y sociales.

Las nuevas tecnologías juegan un papel determinante al potencializar la captación, manipulación, almacenamiento y distribución de la información, para establecer una transición entre una sociedad industrial y otra determinada por una nueva forma de organización económica y social motivada por el desarrollo tecnológico de la información, conocida como sociedad de la información o del conocimiento ${ }^{1}$, provocando con ello modificaciones significativas en los diferentes ámbitos social, político, económico, cultural y educativo que estas involucran. Valenti (2002), citando al Libro Verde sobre la Sociedad de la Información en Portugal, señala que el concepto Sociedad de la Información hace referencia a un modo de desarrollo económico y social que permite adquirir, almacenar, procesar, evaluar, transmitir, distribuir y divulgar la información que se vuelve conocimiento. Su rol es fundamental en la actividad económica que impacta la calidad de vida de las personas.

1 De acuerdo con Burch (2005), el concepto de sociedad del conocimiento «surgió hacia finales de los años 90 y es empleada particularmente en medios académicos, como alternativa de algunos a «sociedad de la información». 
Se hace evidente de esta forma cómo la generación, transmisión y adquisición de conocimientos, gracias a los avances tecnológicos, requiere del replanteamiento de los procesos de enseñanza-aprendizaje de las sociedades de acuerdo con las nuevas circunstancias en que se desarrollan.

Se necesita un cambio en los planteamientos que rigen a los procesos educativos actuales, con el propósito de propiciar realmente la adquisición de la información con miras a la creación de nuevos conocimientos y que, con el apoyo del desarrollo y la aplicación tecnológica, pase: de la estandarización a la personalización, de acuerdo a las necesidades particulares; de la introducción de información a la comprensión y utilización de las capacidades de la inteligencia; de un aprendizaje pasivo dirigido por el profesor a uno activo controlado o compartido con el estudiante; de un aprendizaje descontextualizado a tareas conectadas con la realidad, y de un aprendizaje como un proceso finito a una actividad continua a lo largo de la vida.

En ese sentido, las TIC ofrecen nuevas posibilidades de instrumentación y diversificación de los conocimientos que las tecnologías tradicionales no pueden cubrir. Al respecto, Ferreira (1999) señala que más allá de modernizar lo viejo con los nuevos recursos tecnológicos, el objetivo es innovar a través de un nuevo paradigma pedagógico que en efecto se respalde en ellos para acceder a una formación más integral, de mayor calidad y basado en las demandas del entorno social.

\section{Educación Virtual}

De acuerdo con lo señalado en el sitio web del Ministerio de Educación Nacional de Colombia, actualizado en julio del 2009, la educación virtual o en línea se refiere al establecimiento de programas formativos cuyo escenario de enseñanza y aprendizaje es el ciberespacio. En esta dinámica pierde relevancia la sincronicidad entre tiempo y espacio para permitir que en un ambiente más flexible se propicie el intercambio o la experiencia de aprendizaje. Desde esta perspectiva, las TIC proporcionan nuevas herramientas para la formación e introducen una forma renovada de generar aprendizajes. Así, hoy en día la educación virtual se presenta como una alternativa de educación a distancia prácticamente en todos los niveles de enseñanza, aunque por sus características y particularidades, en el sector de las universidades, donde mayormente se implementa, es el ámbito en el cual se han obtenido los mejores resultados en la dinámica del proceso educativo. 
Se entiende por educación a distancia a la modalidad que ya no requiere del aula ni de profesores presentes físicamente en el proceso. La base es el material escrito que describe programas específicos (Loza, 1997), que se caracterizan por potenciar en los alumnos el aprender a aprender y el aplicar el aprendizaje al mundo real; se otorga, de este modo, la responsabilidad de su aprendizaje al propio alumno.

Para Lorenzo García (citado en Solari y Monge, 2004) la educación a distancia consiste en un sistema apoyado en la tecnología para apoyar el aprendizaje autónomo de los educandos. En él se sustituye la interacción directa de alumnos y profesores, y se ofrecen, por tanto, una serie de recursos didácticos y una acción tutorial.

Según Ávila y Bosco (2001), los ambientes virtuales de aprendizaje no se circunscriben a la educación formal, ni tampoco a una modalidad educativa particular. Se trata de escenarios favorecedores de nuevos conocimientos y experiencias que den lugar al análisis, la reflexión y la apropiación es:

- Espacio de asesoría, en el cual se realizan actividades entre el alumno y el docente para la resolución de dudas y retroalimentación.

- Espacio de experimentación, en el cual, gracias a la implementación de situaciones virtuales, se recrean los procesos educativos simulando situaciones reales.

- Espacio de gestión, en el cual se llevan a cabo los trámites escolares, en general, así como el seguimiento y la evaluación del docente al aprendizaje de sus alumnos. En su versión más actual, la educación se plantea virtual. En ella, la interacción de los actores del proceso ha de permitir una comunicación asincrónica que rompa las barreras de espacio y tiempo.

Las experiencias en esta modalidad son mundialmente recientes. En Estados Unidos, las universidades pioneras fueron, a finales de los años 80 del siglo XX, la Universidad de Phoenix y el Instituto Tecnológico de New Jersey. La gran diferencia entre el desarrollo de los medios tecnológicos en los países del Norte y los que se encuentran en desarrollo, se ha centrado en la disponibilidad y el acceso a estos medios que ha tenido el grueso de su población. 
El Departamento de Educación de los Estados Unidos, en su Plan Nacional de Educación en TIC (NETP) plantea la importancia de implementar un modelo de investigación y desarrollo (I\&D) en educación que se fundamente en escalar las mejores prácticas y las más innovadoras en el uso de las TIC, a la enseñanza y el aprendizaje. De esta manera se busca transferir a la educación las innovaciones tecnológicas existentes y emergentes, apoyando la I\&D para el trabajo a través de organizaciones como la Fundación Nacional para la Ciencia y creando una nueva organización que tenga a su cargo el manejo de los mayores retos en I\&D en el campo de confluencia del aprendizaje de las ciencias, la tecnología y la educación.

El NEPT incide sobre cinco ámbitos:

- Aprendizaje. Se fundamenta en cómo aprender, cuándo y qué se necesita saber. Se proporciona tecnología de punta para posibilitar que todos los estudiantes, sin distinciones y en equidad, accedan a un aprendizaje personalizado, continuo y a lo largo de la vida.

- Evaluación. Se apuntala la necesidad de incorporar nuevas y mejores maneras de diagnosticar fortalezas y debilidades durante el aprendizaje. El propósito es fortalecer el desempeño del alumno y vincular entre sí a grupos de trabajo académico en la dinámica de diseñar, aplicar y utilizar una evaluación con base en las TIC.

- Enseñanza. Apuesta por la interconexión total que provea a los docentes de datos y herramientas de análisis, así como permitirles el acceso a los recursos que les ayuden a actuar con base en los indicios ofrecidos por los datos.

- Infraestructura. Incluye el recurso humano, políticas y modelos sostenibles para el mejoramiento continuo, además de conexión de banda ancha, servidores, software, sistemas de gestión y herramientas administrativas.

- Productividad. Supone la aplicación de la tecnología para propiciar un aprendizaje personalizado y progresivo. También, con el uso de las TIC se pretende planear, dar seguimiento a los gastos y ejercer otras acciones administrativas con el propósito de hacer eficaz el desempeño financiero del sistema educativo. 


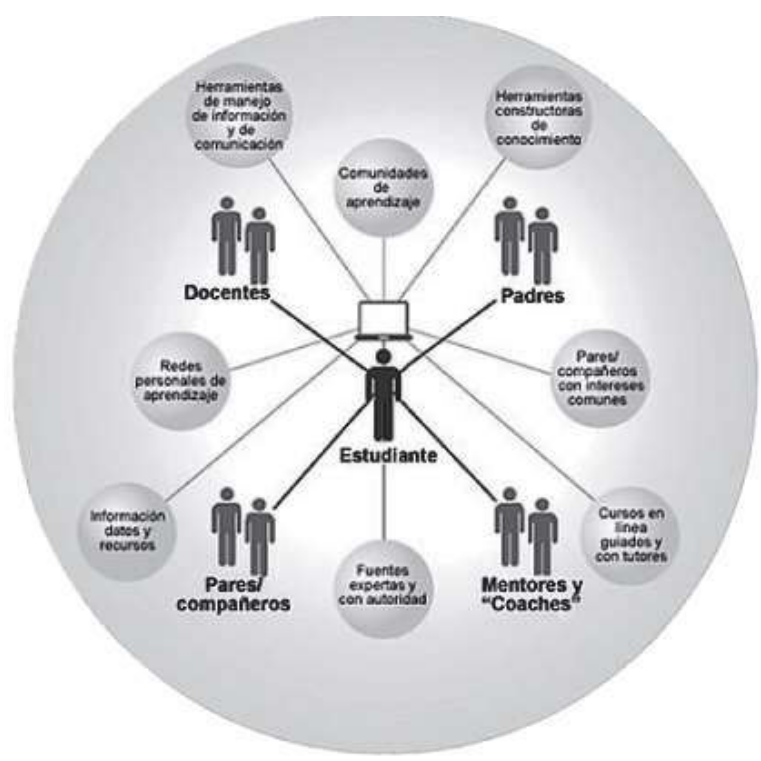

Gráfico: Modelo de Aprendizaje Potenciado por la Tecnología -Elaborado por el Departamento de Educación de los Estados Unidos, en su Plan Nacional de Educación en TIC (NETP, 2010).

Además del Departamento de Educación de los Estados Unidos, otros organismos que han dado cuenta de la relevancia de incorporar en plenitud las TIC y la virtualidad son: la Organización para la Cooperación y el Desarrollo Económico (OCDE), la UNESCO y el Banco Interamericano de Desarrollo (BID).

En sus documentos Sistemas Nacionales de Innovación e Innovación en la Economía del Conocimiento: Implicaciones para la Educación y los Sistemas de Aprendizaje, la OCDE destaca que la fluidez y las sinergias que se generen entre tecnología e información, así como entre la educación y sus resultados investigativos, serán claves para crear un auténtico proceso innovador. Se sustenta que tradicionalmente no se ha dado una real transferencia del conocimiento adquirido hacia la solución efectiva de demandas sociales. Ante ello, propone que el conocimiento científico incorpore prácticas innovadoras y vanguardistas que impacten en los procesos de enseñanza y aprendizaje, y en esa dinámica, la investigación es crucial.

La OCDE también señala la importancia de que los docentes accedan plenamente al uso de redes y comunidades de aprendizaje. El estímulo a la labor docente, indica, es vital. Otro aspecto consiste en optimizar la relación entre unidades descentralizadas de innovación y un sistema global educativo, lo que hará posible desarrollar currículos y contenidos dinámicos y en evolución que rompan la inflexibilidad de ciertas directrices del control

ITINERARIO EDUCATIVO • ISSN OIDI-2753 • AÑO XXVII, N. ${ }^{\circ}$ 6I • ENERO - JUNIO DE 2OI3 • P. 65-88 
central. Finalmente, y de modo esencial, plantea que las TIC han de ser las herramientas que transformarán la educación y las formas convencionales de elaborar las tareas dentro de las instituciones.

\section{Referentes nacionales de innovación}

Entre las entidades educativas que han liderado el fomento de la investigación en Colombia están el Instituto para la Investigación Educativa y el Desarrollo Pedagógico (IDEP) y el Instituto de Tecnologías Abiertas en Educación (ITAE), de la Universidad Pedagógica Nacional de Bogotá. El IDEP describe pautas referidas a la virtualidad, a propósito de la práctica de divulgar sus tareas académicas a lo largo de sus años de existencia; además de que marca como prioridad ejercer las transformaciones tendientes a la innovación considerando la participación y el trabajo colectivo de los profesores.

Por su parte, el ITAE se define como un espacio de indagación, formación y desarrollo de ambientes de aprendizajes bajo la óptica de las TIC. A partir de la transversalidad investigadora entre educación, comunicación, culturas y tecnologías, ofrece asesorías a proyectos institucionales que se basen en el uso de las TIC.

Los posgrados que ofrecen diversas alternativas de educación con sustento tecnológico tienden a ampliar su espectro. El valor que cada vez se impone con mayor frecuencia e interés académico es el de la innovación. La Universidad Pedagógica Nacional tiene una Maestría en Tecnologías de la Información Aplicadas a la Educación, la cual apuesta por la autonomía del estudiante a lo largo de su proceso de aprendizaje, la virtualidad como tendencia que analiza los procesos comunicativos, educativos, sociales y culturales de las comunidades que emplean las modalidades e-learning, b-learning y m-learning, así como las líneas de apoyo tecnológico dirigida hacia grupos que viven con discapacidades. También, la Universidad de los Andes, en su Maestría en Educación, incorpora elementos diversos vinculados a las TIC. Uno muy relevante refiere el análisis crítico y reflexivo acerca del impacto de estas tecnologías en el nivel básico, medio y superior de educación formal y no formal. Otros posgrados con sesgo tecnológico se ofrecen en la Universidad de la Sabana, la Universidad Autónoma de Bucaramanga, y en otras instituciones en las que resulta imperativo formar especialistas que se conciban como recurso humano capaz de interactuar en un medio de enorme competitividad. El objetivo es formar comunidades académicas, científicas y de desarrollo educativo que tengan como referente la contribución que puedan ofrecer a su entorno regional e internacional. 
Hay un compromiso explícito reflejado en múltiples normativas para conducir la tarea educativa en el escenario de la innovación. Los retos son múltiples, pero Colombia se ha de vislumbrar como una de las naciones líderes en la geografía latinoamericana, en tanto sea capaz de hacer pragmáticos los planteamientos legales y tener la capacidad de acceder a la infraestructura tecnológica que permita cumplir los objetivos de generalizar el uso de las TIC, y contribuir con ello a los propósitos de la democratización.

La producción, gestión y uso de recursos educativos digitales constituyen acciones que van a tono con una política de promoción de la industria de contenidos digitales. Al respecto, el Ministerio de Educación Nacional, a través de la Oficina de Innovación Educativa con Uso de TIC y con el respaldo de otras entidades, planteó en el 2011, la Estrategia Nacional de Recursos Educativos Digitales Abiertos dirigidos a instituciones de educación superior. Entre sus propósitos destacan: fortalecer el uso educativo de las TIC, reducir la brecha en el acceso a la información, fomentar la producción de conocimientos en educación, promover la colaboración y cooperación en las comunidades educativas que permita el intercambio de experiencias, colaboración y participación entre sus miembros, motivar la investigación y la innovación educativa, mejorar la calidad de la educación, y promover la producción, gestión y uso de recursos educativos digitales abiertos (MEN, 2012).

Por otra parte, el Sistema Nacional de Recursos Educativos Digitales Abiertos, perteneciente al Ministerio de Educación Nacional, tiene cinco líneas estratégicas, las que en síntesis proponen fortalecer la infraestructura tecnológica, recolectar, organizar, almacenar, catalogar, dar continuidad, publicar, distribuir, buscar, recuperar y desplegar los recursos educativos digitales abiertos, así como desarrollar dinámicas de formación y apropiación en el uso pedagógico de tales recursos (MEN, 2012).

El país asume desafíos irreversibles si se considera que la innovación, vía las TIC, constituye la realidad de un presente que sólo puede mirar al futuro, si se plantea acceder a nuevas modalidades de desarrollo educativo. Todo el entramado social ha sido impactado por la tecnología, y hoy somos testigos de un nuevo modo de vivir y convivir. La educación es el más poderoso caudal de desarrollo para toda nación, y en ella se cifra el verdadero avance. Nuestro país busca alinearse a las corrientes más vanguardistas del desenvolvimiento tecnológico. No puede ser de otro modo, debido a que nuestro real crecimiento se basa en otear 
horizontes de un país más democrático, más equitativo y que genere oportunidades educativas para todas las comunidades, en especial a las históricamente olvidadas. Es un reto colectivo y sólo como sociedad habremos de asumirlo.

\section{Elementos que se deben considerar para el desarrollo de un Ambiente Virtual de Aprendizaje}

Desarrollar ambientes virtuales de aprendizaje resulta un proceso complejo que va más allá de trasladar la clase tradicional a un ambiente virtual. Se requiere que los participantes en el desarrollo de estos ambientes conozcan todos los recursos tecnológicos disponibles (infraestructura, medios, recursos de información, etc.), así como sus ventajas y limitaciones para poder relacionarlos con los objetivos, los contenidos, las estrategias y actividades de aprendizaje y la evaluación.

De acuerdo con Ávila y Bosco (2001) cuando se diseñan ambientes de aprendizaje se han de considerar los requerimientos teóricos, así como las habilidades pedagógicas y técnicas para acceder al aprendizaje y a la socialización del conocimiento a través de la tecnología.

Por ello, como se ha mencionado, el óptimo desarrollo de un AVA implica tomar en cuenta ciertos planteamientos de tipo pedagógico y tecnológico que rijan el proceso, los cuales, por un lado, permitan la correcta estructuración de AVA y, por otro, determinen una mayor calidad y efectividad en los procesos educativos que en ellas se llevan a cabo.

Lo anterior implica revisar las teorías educativas desde una perspectiva apropiada y evaluar las posibilidades que ofrecen los recursos tecnológicos en apoyo al aprendizaje, involucrando en ello según: Nemirovski, Neuhaus y Schlageter (citados por Herrera, 2004, p. 4) los siguientes requerimientos:

- De dominio, referidos a los contenidos emanados de la asignatura misma y que parten de los objetivos de aprendizaje.

- Psicopedagógicos, los correspondientes al enfoque teórico y práctico del aprendizaje de acuerdo con los paradigmas asumidos.

- De interface, los que se derivan de las características propias del medio y el nivel de interactividad que serán utilizados. 
Desde esta perspectiva, resulta necesaria la definición de ciertos criterios que determinen el desarrollo del AVA, tomando en cuenta tres ámbitos del conocimiento:

- Precisión y jerarquización de los contenidos,

- Establecimiento de las estrategias adecuadas para el aprendizaje, $\mathrm{y}$,

- Presentación de la información e implementación de los recursos para ello.

Así los modelos pedagógicos deben estar enfocados a un proceso centrado en el aprendizaje, que permita el desarrollo de valores, actitudes y habilidades requeridos por los alumnos en la sociedad de la información.

Los nuevos paradigmas educativos, centrados en el aprendizaje, situados en un contexto constructivista y basados en conceptos sobre competencias, pueden establecer una planeación didáctica que implique el desarrollo de un diseño pedagógico que, apoyado por la implementación de las TIC, sustente la creación de un modelo didáctico innovador aplicable a los nuevos contextos y espacios para el aprendizaje.

\section{Influencia de las estructuras cognitivas en la construcción de un modelo didáctico para AVA}

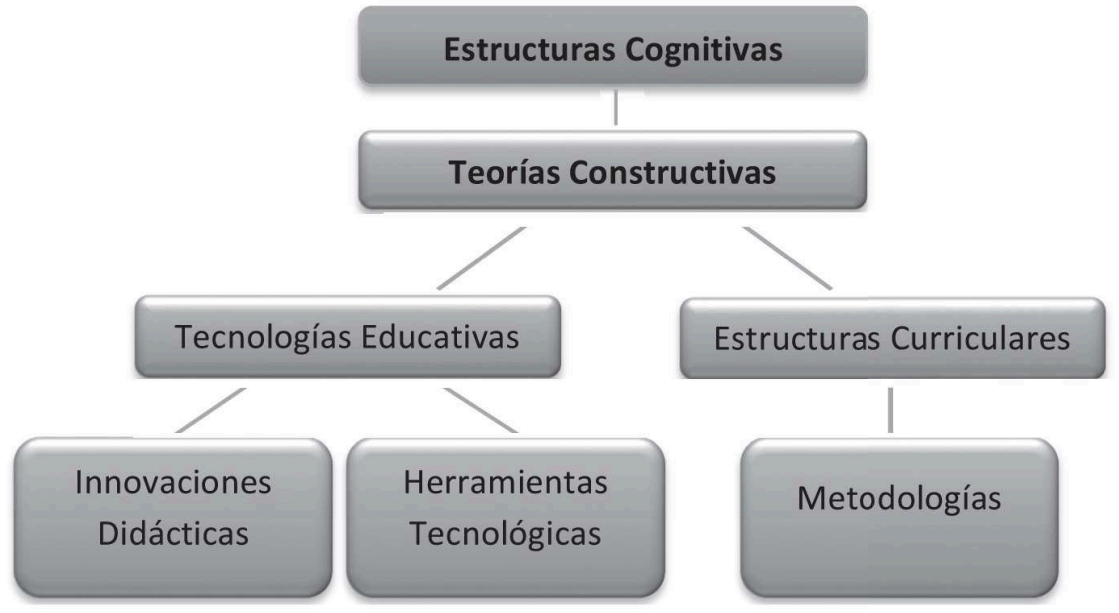

Gráfico elaborado con base en Herrera (2004). Modelo instruccional para el diseño didáctico de ambientes virtuales para el aprendizaje. 
Hablar del uso de las TIC dentro del contexto educativo requiere de la diferenciación entre los conceptos de tecnologías educativas y de tecnologías aplicadas a la educación. Al respecto, Corona et al. (2006) señalan que en la tecnología educativa se implican los medios tecnológicos, la didáctica, la significación cultural y educativa para que en efecto se realice el aprendizaje, mientras que la tecnología aplicada a la educación refiere únicamente las herramientas tecnológicas que, como medios de comunicación vinculados a la enseñanza, no suponen necesariamente una manera distinta o significativa de aprender.

Con base en estas aseveraciones, para esta revisión la planeación didáctica es considerada como una actividad previa al desarrollo del proceso de aprendizaje que permite orientar, de manera coherente, las acciones necesarias para lograr el aprendizaje, involucrando situaciones relacionadas con el diagnóstico de las necesidades educativas, el análisis de la problemática, el diseño y evaluación de las opciones de acción, así como la implantación y evaluación del proceso.

\section{Consideraciones en torno al proceso de aprendizaje}

Antes de proseguir con la descripción del AVA, resulta necesario desarrollar una reflexión sobre el proceso mismo de aprender y las acciones que de él se derivan para comprender los fundamentos de la referida modalidad de ambiente virtual.

Díaz Barriga y Lule (2000) señalan que la planeación didáctica se desarrolla en una serie de pasos, los cuales involucran:

- El diagnóstico, en él se relacionan las necesidades educativas, las condiciones de aprendizaje y los elementos externos que tienen impacto en el proceso educativo;

- La consideración analítica de las características de los problemas, lo que implica un entendimiento integral de la vasta complejidad de la praxis educativa;

- El diseño y la evaluación de las acciones que prevén el resultado de las opciones consideradas, con el propósito de seleccionar la más apropiada para el logro de los objetivos;

- La implantación, que es la puesta en marcha del planeamiento educativo; $y_{\text {, }}$ 
- La evaluación, que plantea balances para analizar el éxito del proceso y sus resultados.

Las circunstancias que definen a las sociedades actuales hacen evidente, de acuerdo con Díaz Barriga y Hernández (2010), la necesidad de favorecer en las personas la autogestión de sus procesos de aprendizaje, desde una postura autónoma y con la disposición del bagaje intelectual que haga posible el aprendizaje continuo a lo largo de la vida.

Esta situación posiciona al aprendizaje en el centro del proceso educativo, y hace necesario con ello entender su significación así como las implicaciones derivadas de su desarrollo.

Al respecto, no existe una apreciación universalmente aceptada sobre lo que es el aprendizaje, sin embargo una definición generalizada del concepto es la que aportan Haseloff y Jorswieck (citados en Küper, 2002) entendido como una variable interviniente que explica adaptaciones a largo plazo a circunstancias externas, a sistemas sociales y a estados internos.

Son varias las posturas que definen el concepto de aprendizaje, las más comunes son las que lo consideran como un cambio duradero en los mecanismos de la conducta a partir de un esquema de estímulorespuesta. Sin embargo, dichas concepciones han sido superadas dando lugar a lo que Ángeles (2003a, pp. 41-42) define como aprendizajeadaptación, entendido como un proceso de cambio que se deriva de la experiencia y de la conservación del entramado organizativo del sistema.

En ese sentido, el aprendizaje consiste en la interacción continua entre el interior y el exterior del sujeto que aprende, tomando en cuenta que la dimensión interior -subjetividad, al asignar significados a los elementos externos mediante un proceso de asimilación, será la que determine el logro del aprendizaje. Sus fases son:

1. Fase de motivación, mediante la cual se genera la expectativa para que el sujeto pueda aprender.

2. Fase de atención y percepción selectiva, la cual modifica el flujo de información que ha llegado al registro sensorial y que pasa a la memoria.

3. Fase de adquisición. En ella se codifica la información que ha entrado en la memoria de corto alcance, transformándola en material simplificado. 
4. Fase de retención, que implica un proceso de acumular la información en la memoria.

5. Fase de recuperación de la información. En ella, la información que ha sido almacenada, en acción a estímulos externos, en la memoria de largo alcance puede ser recuperada.

6. Fase de generalización, mediante la cual el sujeto puede generalizar lo aprendido en nuevas situaciones fuera del contexto original del aprendizaje.

7. Fase de desempeño, a través de la cual la información ya generalizada pasa al generador de respuestas, exhibiendo un desempeño que refleja lo aprendido.

8. Fase de retroalimentación. Se refuerza el desempeño posible gracias a un aprendizaje, confirmándose las expectativas planteadas en la primera fase de este proceso.

La asimilación, de acuerdo con Ángeles (2003a), no debe entenderse como una simple transacción del exterior hacia el interior sino como el resultado de un proceso de elaboración durante el cual el sujeto modifica, en su propio interior, la representación inicial de la realidad externa.

El proceso educativo centrado en el aprendizaje, retomando los planteamientos sugeridos por Ángeles (2003a), establece generalmente tres objetivos concernientes al aprendizaje de los contenidos curriculares, al proceso de aprender a aprender y al desarrollo de la eficiencia y la autonomía de los aprendizajes.

Por un lado, el aprendizaje de contenidos muestra una estrecha relación con el aprendizaje significativo, el cual se produce cuando la nueva información por aprender se relaciona de manera no arbitraria con la estructura cognitiva del alumno.

Dentro de este contexto, se puede definir como estructura cognitiva al repertorio de conceptos e ideas que todo individuo posee en un ámbito del conocimiento, así como su organización.

Por otro lado, el proceso de aprender a aprender se relaciona con la idea de aprender a pensar, potencializando el aprendizaje a partir del desarrollo consciente y autónomo de capacidades y valores por parte del alumno o meta cognición, permitiéndole, a través del mejoramiento de 
técnicas, estrategias y habilidades, acceder al conocimiento y lograr un aprendizaje significativo.

La meta cognición, concepto atribuido a John Flavell, es definida como la capacidad del ser humano de ser consciente de sus procesos y productos cognitivos, la que, de acuerdo Monereo y Castelló (1997), le permite desarrollar conocimiento cada vez más consistente de sus propia competencia y limitaciones.

El término cognición significa, de acuerdo con Rodríguez (2004), razonar el conocimiento alcanzado mediante el ejercicio de las facultades mentales. Así, es común entender a la meta cognición como la cognición de la cognición, o bien como la capacidad de entender, analizar y manejar los propios conocimientos (Ángeles, 2003a).

Finalmente, el último objetivo se relaciona con la posibilidad de impulsar en el alumno la educabilidad cognitiva, entendida como la serie de prácticas, técnicas e instrumentos que asumen como propósito el desarrollo de la eficiencia, el aprendizaje autónomo para desplegar ordenadamente los procesos del pensamiento y de las estructuras mentales del sujeto (Ángeles, 2003b).

Puede apreciarse así la importancia que adquieren las estrategias cognitivas en el desarrollo de procesos educativos centrados en el aprendizaje, desarrolladas bajo diversos enfoques que definen a las teorías cognitivistas y constructivistas, mismas que se describen a continuación.

El aprendizaje ha sido interpretado a lo largo de la historia de muy diversas maneras. Al respecto, Herrera (2004) señala que desde la era Platónica hasta la actualidad se han desarrollado una amplitud de paradigmas del aprendizaje. Con el advenimiento de la psicología se han logrado explicar sistemáticamente estos procesos, lo que ha permitido comprender la profundidad de la mente humana.

El paradigma que ha predominado en las últimas décadas respecto al aprendizaje hace referencia a los dos enfoques: el conductista, que considera al aprendizaje un cambio observable en la conducta del individuo basado en motivaciones extrínsecas y elementales así como en los arreglos ambientales y en la manipulación exterior (Ángeles, 2003a) y el cognitivista, que considera al aprendizaje como un cambio 
interno y sucesivo en las estructuras cognitivas del individuo a través de la experiencia teniendo como base el conocimiento previo (Corral, 1996).

Sin embargo, el aprendizaje considerando los aspectos cognitivos, sociales y afectivos del comportamiento no solo es resultado del ambiente y de las disposiciones internas del individuo, sino más bien de una construcción propia que éste va produciendo día a día derivada de esos dos factores, por lo que puede apreciarse un tercer enfoque el constructivista, que considera al aprendizaje como un proceso de construcción del conocimiento.

El constructivismo surge como una corriente epistemológica preocupada por entender los problemas relacionados con la adquisición del conocimiento, que tiene sustento en autores clásicos que en sus diferentes obras han elaborado la teoría constructivista. Basta con destacar los trabajos de Jean Piaget, Lev Vigotsky, Ernst Von Glaserfeld, Humberto Maturana y David Ausbel, quienes comparten un principio común que considera la relevancia de la acción constructiva del alumno en la elaboración de los aprendizajes, es decir, hay un sujeto cognoscente que con su trabajo constructivo trasciende lo que su entorno le ofrece (Díaz Barriga y Hernández, 2010).

En ese sentido, el análisis de las diferentes teorías que fundamentan al constructivismo lleva a considerar como fuentes principales de la visión constructivista de los procesos de enseñanza-aprendizaje a los planteamientos derivados de la psicología genética de J. Piaget, de las teorías cognoscitivistas del aprendizaje verbal significativo de D. Ausbel y del aprendizaje por descubrimiento propuesto por J. Bruner, así como de la teoría sociocultural inspirada en Vigotsky.

Con base en el planteamiento anterior y retomando a Coll (citado en Díaz Barriga y Hernández, 2010) esta investigación considera que el aprendizaje, desde una concepción constructivista, es el resultado de un intercambio funcional establecido entre el alumno que aprende, el contenido que es objeto de aprendizaje y el profesor que ayuda al alumno a construir significados y a atribuir sentido a lo que aprende.

Esta construcción de conocimientos se producirá cuando el alumno, motivado por una situación de incongruencia entre sus conocimientos y la realidad, interactúe activamente con el objeto de aprendizaje en 
busca de información para la resolución de problemas (Piaget), lo cual le permita establecer, mediante un razonamiento inductivo y mecanismos de descubrimiento (Bruner), una relación significativa entre lo que se le propone y sus saberes previos (Ausbel) en una acción guiada, colaborativa y recíproca (Vigotsky) para lograr nuevos aprendizajes.

Definidos los principales aportes que hace el constructivismo en relación al aprendizaje, cabe señalar que para algunos investigadores como: Newwel, Simon, Vadillo, Kinger, Norman, Cuenca y Mayer este puede ser explicado desde una perspectiva un tanto diferente a las teorías descritas anteriormente, proponiendo una postura epistemológica del aprendizaje fundamentada en la adquisición o desarrollo de competencias por parte del alumno (Herrera, 2004).

Las estructuras cognitivas influyen en el diseño de los modelos didácticos; esta situación ha dado lugar a la definición de una serie de metodologías didácticas tendientes a generar procesos activos de construcción de aprendizaje. Al respecto, Rivera (citado en Benítez, 2010) considera como metodología didáctica a las estrategias que son aplicadas al proceso de enseñanza y aprendizaje, los cuales constituyen el entramado sobre el cual se propicia la instrucción de manera sistemática y fundamentada en teorías del aprendizaje.

\section{Las fases de la modalidad didáctica AVA}

Retomando los planteamientos de Sierra (2006), Mc Griff (2000), Martínez (2009), Ochoa (2006), Esteller y Medina (2009), se pueden mencionar varias metodologías de diseño instruccional. La que ahora nos ocupa es la metodología de diseño didáctico en Ambientes Virtuales de EnseñanzaAprendizaje (AVEA), que aborda el diseño de cursos virtuales a partir de la redimensión de los nexos entre las intenciones y exigencias educativas y las potencialidades pedagógicas, tecnológicas y organizacionales de los AVA. Se lleva a cabo a través de las siguientes fases:

1. Fase de Planificación del proceso de diseño didáctico, orientada a crear las condiciones necesarias para la realización del curso así como su adecuación a situaciones concretas, asegurando la viabilidad del mismo.

2. Fase de Modelación del proceso educativo y su ambiente virtual, que comprende la elaboración del programa de estudio,

ITINERARIO EDUCATIVO • ISSN OILI-2753 • AÑO XXVII, N. ${ }^{\circ}$ 6I • ENERO - JUNIO DE ZOI3 • P. 65-88 
la preparación del diagnóstico y la modelación de los espacios del ambiente virtual.

3. Fase de Control y evaluación del proceso de diseño didáctico, mediante la cual dar seguimiento al proceso de diseño del curso.

4. Fase de Validación del proceso de diseño didáctico y sus resultados, que abarca la revisión integral del curso por parte de un equipo de experto.

La modalidad AVA tiene como bastión de desarrollo la tecnología. En una aproximación asertiva al proceso de centrar la práctica educativa en el aprendizaje, el ambiente virtual ofrece expectativas que permitan democratizar el acceso al conocimiento. En ello radica su fuerza y la importancia de alentar su crecimiento y su presencia como modalidad académica que mira al futuro, con la orientación de fomentar la adquisición de un conocimiento que trascienda limitaciones temporales o espaciales. De manera óptima, el AVA habrá de generalizarse con la perspectiva de generar competencias que influyan en el logro del bienestar ciudadano.

\section{Reflexiones finales sobre el uso de la tecnología en el ámbito educativo}

- La tecnología digital y con ella la informatización y el mundo de los computadores personales, así como la aportación de las telecomunicaciones a la difusión y transporte de información han iniciado ciertos cambios substanciales en el proceso comunicativo. Hoy todos estos sistemas para la comunicación convergen utilizando un solo medio, lo que hace que una persona o grupo pueda aprovechar todos los medios de comunicación prácticamente al mismo tiempo. Estamos hablando de las redes de información y más concretamente del Internet. Cuando se utiliza un sistema informático como herramienta educativa, pueden darse varios fenómenos:

- La actividad del alumno está controlada por el computador y la estrategia pedagógica utilizada es de tipo tutorial. Se basan en los principios de los Modelos Lingüísticos del procesamiento de la información e incorpora ideas procedentes del programa cognitivo.

- El computador se utiliza con un entorno simulado, un micromundo, sometido a sus propias leyes y que el alumno 
debe descubrir o aprender a utilizar mediante la exploración y la experimentación dentro de ese entorno. El sistema informático se convierte en una herramienta que potencia el desarrollo de las habilidades cognitivas del alumno.

- No se intenta inducir en el alumno los procesos rigurosamente planificados sino que existe un ambiente inteligente que es capaz de simular alguna de las capacidades cognitivas del alumno y utilizar los resultados de esta simulación como base de las decisiones pedagógicas a tomar. La innovación técnica y sus repercusiones en nuevas formas expresivas y modos de comunicación plantean un reto al sistema educativo para desarrollar nuevas estrategias de selección y análisis de los conocimientos que se construyen o reconstruyen. Nos encontramos cada vez más con alumnos que saben acceder de forma rápida a grandes cantidades de información y alumnos acostumbrados a un tipo e lenguaje más global; dado que la tendencia convergente de tecnologías de la imagen, sonido y escritura desemboca en una integración de los tres subsistemas expresivos (Audio-escritovisualidad).

- En definitiva, el desarrollo tecnológico ha provocado el desarrollo de nuevas habilidades en los estudiantes que deben ser tenidas en cuenta. Esta situación puede cuestionar seriamente los métodos pedagógicos tradicionales más limitados en las fuentes de datos, de carácter discursivo y menos atractivos para motivar a los alumnos.

- Con todo esto se abren las puertas para desarrollar ideas acerca de enseñar en el dominio de los sistemas multimediales y de las pedagogías virtuales. Es francamente posible diseñar estrategias para enseñar a investigar usando estos contextos de sofisticada tecnología

Por lo anterior, la creciente incursión de las TIC en los procesos educativos, definida dentro del contexto de la tecnología educativa, está provocando que las teorías de diseño de los modelos didácticos presenten una nueva forma de conceptualización, de cara a la creación de nuevos ambientes de aprendizaje.

Actualmente se han realizado esfuerzos significativos para poder definir las teorías y los modelos educativos adecuados para el desarrollo de 
Ambientes Virtuales de Aprendizaje, los que determinen las pautas para la planificación, el desarrollarlo y la aplicación de situaciones didácticas en línea.

\section{Conclusión}

\section{Las estructuras cognitivas y el estatus del cambio de creencia}

El discernimiento entre el tipo de implicación dada entre las tecnologías E-learning y los procesos cognitivos implícitos en el aprendizaje como propiedad humana no resulta más esclarecedor si sólo se subraya la presencia secuencial de tareas y procesos mecánicos o automatizados. Por ejemplo: la fase de motivación como detonante de la expectativa puede ser un hecho meramente circunstancial sino no es instanciado propiamente como una parte importante de la estructura cognoscitiva. Si es visto de esta manera, es decir, como parte del sistema cognitivo, la motivación juega un rol intrínseco al sistema y no es un apéndice de éste. Dicho rol permite medir el cambio de actitud frente al conocimiento, de tal modo que la motivación es más un detonante para preparar al conocimiento que un estado psicológico influido por la presencia de condiciones controladas del ambiente.

La atención y percepción selectiva, por otro lado, son los dispositivos cognitivos más apropiados para fijar los objetos o eventos cognoscibles en un espacio de interpretación simbólica. La atención fija el objeto no solo como producto de la experiencia, sino que también lo capta por medio de un sistema selectivo de reglas, las cuales permiten su abstracción. La percepción es el resultado de la singularidad del objeto, en la cual además de identificarlo le atribuimos atributos. Así, en los AVA los dispositivos cognitivos de atención y percepción son codificaciones de la singularidad de los objetos cognoscibles, pues ellos ya son objetos cargados de significado.

Luego de que esta simbolización se fije en la memoria, lo que comúnmente se llama «adquisición», sufre un proceso de codificación expresable o traducible lingüísticamente, lo que determina la relación lenguajepensamiento. Aunque el conocimiento de estos procesos permanece oscuro, lo que sabemos de ello nos permite inquirir en la idea de cierto estatus de la información o cierto estatus de creencia. La adquisición, 
entonces, no es el fijar o tallar la memoria con símbolos, es más bien, el paso del símbolo-imagen al símbolo-lenguaje.

El olvido aún es un tema poco conocido y explicado, el papel de la retención no es el de explicarlo, sino el de señalar cómo del símbolo a la imagen y de la imagen al código hay información que persiste. La persistencia de esta información es otra clave que posibilita la conexión entre los dispositivos virtuales y las estructuras cognitivas. Los modelos automáticos de aprendizaje son un ejemplo de dicha conexión, algunos de estos modelos se sustentan en el aprendizaje por refuerzo, donde éste es entendido como persistencia y no como rutina. De esta manera el papel de la memoria es otro, no el de simplemente recordar o el de fijar información, es del de hacer selectiva la información que persiste y con ella los procesos implícitos en el procesamiento de la información.

La generalización como uno de los dispositivos cognitivos ejecutivos es quizá el que ofrece mayor interés en el estudio de los modelos AVA. De hecho en la generalización es observable el estatus del cambio de creencia, pues aporta información nueva y concluyente que es producto de la funcionalidad de otros dispositivos cognitivos contingentes. La estructural cognitivas otorga a la generalización un papel medular puesto que su función se centra en resolver los estados de duda, que a su vez son originados por anomalías o desequilibrios cognitivos. En los AVA la generalización es un procedimiento inductivo, que muchas veces no es explícito. La generalización se transforma en un recurso de aprendizaje, en un instrumento si se quiere, pero su función no es medular. En este sentido, los AVA son recursos incompletos y en algunos casos no muy bien interpretados, pues no se explora de manera adecuada el vínculo necesario y axiomático entre estructuras cognitivas y los procesos virtuales como requisito para liberar y producir estados de creencia como objetivos del aprendizaje.

Finalmente, para acotar este estudio, es importante señalar que las evidencias para mostrar los desplazamientos señalados párrafos arriba no pueden surgir, en las condiciones actuales, de otro lugar que del estudio del lenguaje mismo. El lenguaje se constituye en el fundamento empírico que permite al investigador sopesar el rendimiento no solo de la instrumentación tecnológica aplicada al aprendizaje, sino que ciertamente provee del soporte material para dar cuenta de los procesos implícitos mentales como acaecimientos o datos para ejemplificar una estructura cognitiva coherente. 


\section{Referencias}

Ángeles, O. (2003a). El proceso educativo desde los enfoques centrados en el aprendizaje. Recuperado el 15 de marzo de 2013, de: http://www. lie.upn.mx/docs/docinteres/EnfoquesyModelosEducativos2.pdf

Ángeles, O. (2003b). Fundamentos psicopedagógicos de los enfoques y estrategias centrados en el aprendizaje en el nivel de educación superior. Recuperado el 10 de marzo de 2013, de: http://www.lie.upn. $\mathrm{mx} /$ docs/docinteres/EnfoquesyModelosEducativos1.pdf

Ávila, P. y Bosco M. (2001). Ambientes Virtuales de Aprendizaje. Una nueva experiencia. Recuperado el 13 de octubre del 2012, de la Unidad de Investigación y Modelos Educativos del Instituto Latinoamericano de la Comunicación Educativa: http://investigacion.ilce.edu.mx/ panel_control/doc/c37ambientes.pdf

Benítez, M. (2010). El modelo de diseño instruccional ASSURE aplicado a la educación a distancia. Recuperado el 12 de mayo de 2013, de Revista Académica de Investigación: http://www.eumed.net/rev/ tlatemoani/01/pdf/63-

Corona, L., et. al. (2006). Tecnología educativa o Nuevas tecnologías en la educación. Revista Cognición, No. 8. (Diciembre). México.

Corral, R. (1996). La pedagogía cognoscitiva. Colombia: Poira Editores.

Díaz Barriga, F. y Hernández, G. (2010). Estrategias docentes para un aprendizaje significativo. Una interpretación constructivista. México: Mc Graw Hill.

Díaz Barriga, F. y Lule, M. (2000). Metodología de diseño curricular para educación superior. México: Trillas.

Esteller, V., \& E., M. (2009). Evaluación de 4 modelos instruccionales para la aplicación de una estrategia didáctica en el contexto de la tecnología. Recuperado el 18 de Mayo de 2013, de Revista de tecnología de información y comunicación en educación: http:// servicio.bc.uc.edu.ve/educacion/eduweb/vol3n1/art5.pdf

Ferreira, R. (1999). Hacia nuevos ambientes de aprendizaje. Red escolar SEP-ILCE. Recuperado el 23 de febrero de 2013, dehttp://redescolar. ilce.edu.mx/redescolar/lecturas_BB/nuevos_ambientes.pdf

García, F. (2004). La tesis y el trabajo de tesis. México: Limusa. 
Ginés, J. (2005) La necesidad del cambio educativo para la sociedad del conocimiento. En Revista Iberoamericana de Educación, No. 35 (mayo-agosto). OEI.

Herrera, M. A. (2004). Las nuevas tecnologías en el aprendizaje constructivo. En Revista Iberoamericana de Educación, 34/4.

Kuper, H. (2002). Stichwort: Qualität im Bildungssystem. En Zeitschrift für Erziehungswissenschaft. 5. Agno, Número 4/2002

Loza, J. (1997). Notas sobre Educación Continua Abierta y a Distancia. En Educación Superior Anuies , 71-73.

Martínez, A. C. (2009). Investigación documental: El diseño instruccional en la educación a distancia. Un acercamiento a los modelos. Revista de innovación educativa, 6 (10).

Mc Griff, S. (2000). Instructional Systems. Recuperado el 2013 de Mayo de 2013, de College of Education, Penn State University: http:// disenoinstrucional.files.wordpress.com/2007/09/addiemodel.doc

Monereo, C. y Castelló, M. (1997). Las estrategias de aprendizaje. Cómo incorporarlas a la práctica educativa. Barcelona. Ed. Edebé

MEN (2009). Educación virtual o en línea, recuperado el 8 de junio de 2013, de http://www.mineducacion.gov.co/1621/article-196492.html

MEN (2012). Recursos Educativos Digitales Abiertos. Bogotá D.C., Cundinamarca, Colombia: Graficando Servicios Integrados. Recuperado el 6 de abril de 2013, de http://www.colombiaaprende. edu.co/reda/REDA2012.pdf

NETP. (2010). Transforming American Education, Learning Powered by Technology. Washington: U.S. Departament of Education Office of Educational.

Ochoa, E. (. (2006). Una concepción desarrolladora para el diseño didáctico de cursos de superación a distancia en ambientes virtuales de enseñanza-aprendizaje. Recuperado el 16 de Mayo de 2013, de Entornos de aprendizaje virtual como medios de inclusión. III Congreso on line http://www.cibersociedad.net/congres2006/gts/ comunicacio.php?id=363

Rodríguez, L. (2004). La teoría del aprendizaje significativo. Centro de educación a distancia (C.D.A.D.). Santa Cruz de Tenerife. 
Sierra, H. (2006). Modelo Pedagógico Humanista Tecnológico de la Dirección Nacional de Servicios Académicos Virtuales. Obtenido de Dirección Nacional de Innovación Académica, UN Virtual: http:// www.virtual.unal.ed

Solari, A., y Monge, G. (4 de Abril de 2004). Primer Congreso Virtual Latinoamericano de Educación a Distancia. Recuperado el 16 de mayo de 2013, de Latín Educa 2004.com.: http://www.ateneonline. net/datos/96_03_Birri_Roberto.pdf

Valenti, P. (2002). La Sociedad de la Información en América Latina y el Caribe: Tics y un nuevo Marco Institucional. Revista Iberoamericana de Ciencia, Tecnología, Sociedad e Innovación, Organización de estados iberoamericanos para la Educación, la Ciencia y la Cultura. No. 2. (Enero-Abril). 